\title{
FORMULATION AND EVALUATION OF GASTRORETENTIVE MICROBALLOONS OF ACEBROPHYLLINE FOR THE TREATMENT OF BRONCHIAL ASTHMA
}

\author{
BHUVANESWARI ${ }^{1 *}$, MANIVANNAN ${ }^{2}$, AKSHAY M $^{3}$, NIFY F ${ }^{4}$
}

${ }^{1}$ Department of Pharmaceutics, Periyar College of Pharmaceutical Sciences, Tiruchirappalli - 620 021, Tamil Nadu, India. ${ }^{2}$ Department of Biotechnology, Bharath College of Science and Management - 613 005, Thanjavur, Tamil Nadu, India. ${ }^{3}$ Department of Pharmaceutical Sciences, Chemist College of Pharmaceutical Sciences and Research, Ernakulam - 682 308, Kerala, India. ${ }^{4}$ Department of Pharmacology, RVS College of Pharmaceutical Sciences, Coimbatore - 641 402, Tamil Nadu, India. Email: bhuvanpharm@gmail.com

Received: 05 May 2016, Revised and Accepted: 12 May 2016

\section{ABSTRACT}

Objective: Gastroretentive dosage forms are an approach for prolonged and predictable drug delivery in the upper gastrointestinal tract to control the gastric residence time. Microballoons are considered as one of the most promising buoyant drug delivery systems as they possess the advantages of both multiple-unit systems and good floating properties. Acebrophylline is a xanthine derivative with potent bronchodilator, mucosecretolytic, and anti-inflammatory property. It is used to treat bronchial asthma and chronic obstructive pulmonary diseases.

Methods: Microballoons of acebrophylline were prepared by emulsion solvent diffusion method using hydroxypropyl methylcellulose (HPMC) and ethyl cellulose (EC) as polymer. The microballoons were evaluated with their micromeritic properties, particle size, tapped density, compressibility index, angle of repose, percentage yield, in vitro buoyancy, entrapment efficiency, drug-polymer compatibility, scanning electron microscopy (SEM), and drug release kinetics.

Results: The mean particle size of the microballoons formulation MB1 to MB6 containing HPMC and EC was in the range between $226 \pm 16$ and $577 \pm 10$, respectively. The mean particle size of microballoons was found to increase with increasing polymer concentration. The micromeritic properties were found be good, and SEM confirmed their hollow structure with smooth and dense which helped to prolong floating to increase residence time in stomach. The in vitro drug release studies showed controlled release of acebrophylline microballoons in the simulated gastric fluid more than 12 hrs.

Conclusions: The results showed that the prepared floating microballoons of acebrophylline prove to be potential multiple-unit delivery devices adaptable for safe and effective sustained drug delivery.

Keywords: Microballoons, Acebrophylline, Bronchial asthma, Hydroxypropyl methylcellulose, Ethyl cellulose.

(C) 2016 The Authors. Published by Innovare Academic Sciences Pvt Ltd. This is an open access article under the CC BY license (http://creativecommons. org/licenses/by/4. 0/) DOI: http://dx.doi.org/10.22159/ajpcr.2016.v9i5.12603

\section{INTRODUCTION}

The oral drug administration is the most predominant route for drug delivery due to ease of dosing administration, patient compliance, and flexibility in formulation. Effective oral drug delivery process depends on the factors such as gastric emptying process, gastrointestinal transit time of dosage form, drug release from the dosage form, and site of absorption of drugs. Oral controlled release drug deliveries have recently been of increasing interest in the pharmaceutical field to achieve improved therapeutic advantages. It is primarily aimed at achieving more predictable to control the drug release, and increased bioavailability, thereby obtaining a maximum therapeutic effect [1]

Gastroretentive dosage forms (GRDFs) are a drug delivery formulation that is designed to remain in the gastric region for a long period and significantly prolong the gastric retention time (GRT) of drugs. This technology has generated enormous attention over the last few decades owing to its potential application to improve the oral delivery of some important drugs. GRDFs prolonged retention in the upper gastrointestinal tract, greatly improve their oral bioavailability and/or their therapeutic outcome [2]. Various techniques were used to encourage gastric retention of an oral dosage form. A significant approach for the treatment of gastric disorders can be achieved by floating drug delivery systems (FDDS). FDDS have a bulk density less than gastric fluids; they can float on the gastric juice in the stomach; the drug is released slowly at the desired rate from the system [3]. A number of FDDS involving various technologies have been developed such as single and multiple-unit hydrodynamically balanced systems, single and multiple-unit gas generating systems, hollow microspheres, and raft forming systems [4].
Hollow microspheres/microballoons are considered as one of the most promising buoyant systems. They possess the unique advantages of multiple-unit systems as well as better floating properties because of central hollow space inside the microsphere. The drug release and betterfloating properties mainly depend on the type of polymer, plasticizer, and the solvents used for formulation. Commonly used polymers such as polycarbonate, cellulose acetate, calcium alginate, Eudragit $\mathrm{S}$, agar and low methoxylated pectin were used in the preparation of hollow microspheres [5]. Hollow microspheres loaded with drugs in their outer polymer shell were prepared by simple solvent evaporation or solvent diffusion evaporation method to prolong the GRT of the dosage form. The microballoons floated continuously over the surface of an acidic dissolution media containing a surfactant for more than 12 hrs [6].

Asthma is the most common chronic disease among children and also affects millions of adults. About 235 million people around the globe suffer from non-communicable diseases, and this number is rising particularly in the low- and middle-income countries as they become more urbanized. Asthma can also be fatal and causes an estimated 250,000 deaths annually. Bronchial asthma is common asthma, which is a chronic inflammatory disease of the lower respiratory tract. It is characterized by non-specific bronchial hyper responsiveness, which may lead to reversible airway inflammation, mucus production, and variable airflow obstruction. However, recent research mainly focuses to identify the most effective drug therapy and therapeutic targets [7].

Acebrophylline, chemically designed as ambroxol theophylline-7acetate, is a compound with potent bronchodilator, mucosecretolytic, and anti-inflammatory activity. It is used to treat bronchial asthma and chronic obstructive pulmonary disease [8]. Acebrophylline inhibits 
phospholipase A and phosphatidylcholine leading to lesser production of the powerful pro-inflammatory substances such as leukotrienes and tumor necrosis factor. By inhibiting the synthesis and release of these inflammatory mediators, acebrophylline reduces inflammation, a key factor in airway obstruction, especially in chronic forms [9]. Acebrophylline is therapeutically effective in patients with acute or chronic bronchitis; it reduces the frequency of episodes of bronchial obstruction and reduces the need for B2-agonists and improves indexes of the ventilator. However, drug shows site-specific absorption in the intestine. Standard immediate release marketed tablets of acebrophylline used to treat nocturnal bronchial asthma when taken 2 tablets 4 times daily are chemically equivalent to $4 \mathrm{~g}$ daily dose. It improves the therapeutic efficacy by reducing the dosing frequency and benefits, especially in the case of patients with nocturnal bronchial asthma [10]. The objective of the present study is to design, formulate, and evaluate gastroretentive drug delivery system of acebrophylline using hydroxypropyl methylcellulose (HPMC) and ethyl cellulose (EC) at different ratios for the treatment of bronchial asthma.

\section{METHODS}

\section{Chemicals}

Acebrophylline was received as gratis sample from Sunglow Pharmaceuticals Pvt. Ltd., Pondicherry, India; HPMC Methocel E50 was obtained from Hi Media Laboratories Ltd., Mumbai, India; EC was purchased from Loba Chem Pvt. Ltd., Mumbai, India; dichloromethane (DEM) and ethanol were obtained from E. Merck India Ltd, Mumbai. All other chemicals, reagents, and solvents used were of analytical grade.

\section{Formulation of acebrophylline microballoon}

Floating microballoons containing acebrophylline with a central hollow cavity were prepared by the solvent evaporation technique [11]. Weighed quantities of acebrophylline, HPMC, and EC were dissolved in a mixture of ethanol and DEM (1:1 solvent ratio) at room temperature. The polymer solution was poured into $250 \mathrm{~mL}$ distilled water containing $0.01 \%$ Tween 80 and the resulting solution was stirred with a propeller-type agitator at $300 \mathrm{rpm}$ and $40^{\circ} \mathrm{C}$ for $1 \mathrm{hr}$ to allow the volatile solvent to evaporate. The finely developed microballoons were then filtered, washed with distilled water, and dried in vacuum. The different ratios of polymers were used to prepare the microballoons. The various formulations are tabulated (Table 1).

Drug-excipient compatibility studies by Fourier transform infrared (FT-IR) analysis

The FT-IR spectroscopy analysis was conducted for the analysis of drugpolymer interaction and stability of drug during formulation process [12]. About 2-3 mg of samples was mixed with dried potassium bromide of equal weight and compressed to form a $\mathrm{KBr}$ disc. FT-IR spectrums of pure acebrophylline, HPMC, EC, and floating microballoons (formulation) were recorded using FT-IR spectrophotometer (Shimadzu 1700 S, Japan).

\section{Characterization of microballoons}

\section{Micromeritic properties}

The microballoons were characterized by their micromeritic properties such as particle size, Bulk density, tapped density, \% compressibility index, and flow properties such as angle of repose [13].

\section{Table 1: Composition of floating microballoons formulations}

\begin{tabular}{llll}
\hline $\begin{array}{l}\text { Formulation } \\
\text { code }\end{array}$ & $\begin{array}{l}\text { Drug: } \\
\text { Polymer ratio } \\
\text { (HPMC/EC) }\end{array}$ & $\begin{array}{l}\text { Temperature } \\
\left({ }^{\circ} \mathbf{C}\right)\end{array}$ & $\begin{array}{l}\text { Solvent ratio } \\
\text { ethanol/DCM }\end{array}$ \\
\hline MB1 & $1: 2: 1$ & $30-40$ & $1: 1$ \\
MB2 & $1: 3: 1$ & $30-40$ & $1: 1$ \\
MB3 & $1: 4: 1$ & $30-40$ & $1: 1$ \\
MB4 & $1: 1: 2$ & $30-40$ & $1: 1$ \\
MB5 & $1: 1: 3$ & $30-40$ & $1: 1$ \\
MB6 & $1: 1: 4$ & $30-40$ & $1: 1$ \\
\hline
\end{tabular}

HPMC: Hydroxypropyl methylcellulose, EC: Ethyl cellulose

\section{Particle size}

The particle size analysis of microballoons was determined with an optical microscopic method [14]. The particle size of the prepared microballoons was dispersed in glycerin, and a drop of the above dispersion was transferred on a glass slide and observed under an optical microscope under regular polarized light, and the mean particle size was calculated by measuring 100 microballoons $(n=3)$ with the help of a calibrated eyepiece micrometer and stage micrometer. The average diameter was calculated using the following formula:

Average diameter $=\frac{\text { Total diameter of microballoons }}{\text { Number of microballoons }} \times$ Calibration factor

\section{Bulk density/fluff density}

It is the ratio between a given mass of a powder and its bulk volume. Initial density also is known as fluff or poured bulk density. Bulk density of the formulated microballoons was determined by taking a known quantity about $2 \mathrm{~g}$ of formulated microballoons in a clean measuring cylinder, and initial volume was measured. The bulk density was calculated by the following equation:

Bulkdensity $=\frac{\text { Mass of microballoons in grams }}{\text { Volume of microballoons in } \mathrm{cm}^{3}}$

\section{Tapped density}

Tapped density of microballoons was done by the tapping method [15]. Formulated microballoons $(2 \mathrm{~g})$ were transferred into $10 \mathrm{~mL}$ measuring cylinder. After observing the initial volume of microballoons, the tapping was continued on a hard surface until no further change in volume was calculated using tapped density apparatus (Pharma Chem Machineries, model C-BD 100). The tapped density was calculated according to the following formula:

Tapped density $=\frac{\text { Mass of microballoons in grams }}{\text { Volume of microballoons after tapping in } \mathrm{cm}^{3}}$

\section{Compressibility index}

The percentage compressibility of powder is a direct measure of the potential powder arch or bridge strength and stability and also called as Carr's index and is computed according to the following equation:

$\%$ Compressibility index $=\frac{\text { Tapped density }- \text { Fluff density }}{\text { Tapped density }} \times 100$

Angle of repose

The flow property of floating microballoons is usually assessed by determining the angle of repose. The angle of repose of microballoons was determined by fixed funnel method [16]. The microballoons were allowed to fall freely through a funnel until apex of conical pile just touched the tip of the funnel. The angle of repose $\varnothing$ was determined according to the following formula:

$$
\begin{aligned}
& \text { Angle of repose }(\varnothing)=\tan ^{-1} \quad \text { Height of the pile }(\mathrm{h}) \\
& \text { Radius of the base of the pile formed by } \\
& \text { the floating microballoons }(r)
\end{aligned}
$$

\section{Percentage yield}

Percentage yield of floating microballoon formulation was determined by weighing after drying [17]. The actual weight of microballoons was divided by the total weight of all the non-volatile components used for the preparation of microballoons and is represented by the following formula:

$$
\text { Percentage yield }=\frac{\text { Actual weight of floating microballoons }}{\text { Total weight of excipients and drug }} \times 100
$$

\section{Entrapment efficiency}

The floating microballoons (10 mg) were taken and dissolved in $10 \mathrm{~mL}$ of ethanol and volume was made up with $0.1 \mathrm{~N} \mathrm{HCl}$. The solution was filtered and analyzed spectrophotometrically (Shimadzu UV-1700 S Japan) at $252 \mathrm{~nm}$ using the calibration curve. Each batch should be 
examined for drug content in a triplicate manner. The entrapment efficiency of floating microballoons can be calculated by the following formula:

$$
\text { Entrapment efficiency }=\frac{\text { Actual drug content }}{\text { Theoretical drug content }} \times 100
$$

\section{In vitro buoyancy}

Microballoons equivalent to $100 \mathrm{mg}$ were spread over the surface of a USP XXIV Dissolution apparatus (Type II) filled with $900 \mathrm{~mL}$ of 0.1 $\mathrm{N} \mathrm{HCl}$ fluid (pH 1.2) containing Tween $80(0.02 \mathrm{w} / \mathrm{v} \%)$ to stimulate gastric fluid at $37^{\circ} \mathrm{C}$ [18]. The medium was agitated with a paddle rotating at $100 \mathrm{rpm}$ at $37 \pm 0.5^{\circ} \mathrm{C}$ for $12 \mathrm{hrs}$. After $12 \mathrm{hrs}$, the floating and settled portions of microballoons were recovered separately. The microballoons were dried and weighed. The buoyancy percentage of microballoons was calculated by the following equation:

$$
\text { Buoyancy }(\%)=\frac{\text { Floating microballoons }}{\text { Total mass of microballoons }} \times 100
$$

\section{Surface morphology}

The surface morphology of microballoons was investigated using scanning electron microscopy (SEM) [19]. The samples for SEM were prepared by lightly sprinkling the microballoons powder on a double adhesive tape which stuck to a stub. The stubs were then coated with platinum under an argon atmosphere using a gold sputter module in a high vacuum evaporator. The samples were then randomly scanned, and photomicrographs were taken on higher magnification $(\times 500)$ for surface morphology.

\section{In vitro drug release}

The release rate of drug from microballoons was determined using USP dissolution testing apparatus Type II (paddle type). The dissolution test was performed using $900 \mathrm{ml}$ of $0.1 \mathrm{~N} \mathrm{HCl}$, at $37 \pm 0.5^{\circ} \mathrm{C}$ and $100 \mathrm{rpm}$. Microballoons equivalent to $100 \mathrm{mg}$ of acebrophylline were used for the test. A $5 \mathrm{ml}$ sample solution was withdrawn from the dissolution apparatus for $1 \mathrm{hr}$, and thereafter every $1 \mathrm{hr}$ up to $12 \mathrm{hrs}$. Samples were replaced an equal amount of fresh dissolution medium $0.1 \mathrm{~N}$ $\mathrm{HCl})$ to maintain sink conditions. All experiments were performed in triplicate. The samples were filtered through Whatman filter paper and were analyzed at $252 \mathrm{~nm}$ by an ultraviolet spectrophotometer. The drug release data were fitted to zero order (cumulative \% drug release versus time), first order (log cumulative \% drug retained versus time), and Higuchi models (cumulative \% drug released versus square root of time) to assess the kinetics of drug release and determine the release mechanism of the drug from the floating microballoons [20].

\section{Accelerated stability studies}

Drug decomposition or degradation occurs during storage, because of chemical alteration of the active ingredients or due to product instability, leading to lower concentration of the drug in the dosage form; hence, the stability of pharmaceutical preparation need to be evaluated. Stability studies were carried out at $40 \pm 2^{\circ} \mathrm{C}$ and $75 \pm 5 \%$ relative humidity for 90 days.

\section{Statistical analysis}

Experimental results were expressed as mean \pm SEM, $n=3$.

\section{RESULTS AND DISCUSSION}

\section{Formulation of acebrophylline microballoons}

The floating microballoons were prepared by emulsion solvent diffusion evaporation method using combination of different ratio of polymers HPMC and EC (Table 1). The pharmaceutical development studies have to be carried out with the purpose of selecting right dosage form and a stable formulation. These studies give detailed description of all the steps involved in the process of development of the finished procedure. Such details are intended toward identifying critical parameters involved in the process, which have to be controlled to give reliable and reproducible quality product [21].

Different formulations were formulated and studied for their physicochemical characterization. The formulations of microballoons were prepared based on the formula in which different percentage of polymer, i.e., by gradually increasing the EC concentration in combination with a fixed concentration of HPMC and also by gradually increasing the HPMC concentration in combination with a fixed concentration of EC. In this process, the diffusion of ethanol precedes the evaporation of DEM from the droplet into the aqueous medium drastically reducing the solubility of polymer in the droplet and forming a gel-like film on the surface. The mechanically strong solidified film produced at the surface of droplet with further depletion of ethanol prevented rupture and shrinkage of microspheres during the evaporation of DEM from the droplets. The cavity produced by gas phase was gradually filled with water due to the reduced pressure inside the droplet that was caused by evaporation of DEM. The microballoons were prepared by removing water from the cavity of the microsphere with air drying. The presence of Tween 80 prevents aggregation of droplets, as it acts as an emulsifying agent. The rapid removal of the solvent leads to the formation of microballoons of acebrophylline, which prolongs the GRT of the dosage form for $12 \mathrm{hrs}$ [22].

\section{FT-IR spectra analysis of drug and polymer compatibility}

The compatibility between the drug and the polymer were evaluated using FT-IR peak matching method. The characteristic peaks $\left(\mathrm{cm}^{-1}\right)$ due to pure acebrophylline at $3475.93,2975.32,1638.42,1457.49$, $1311.50,879.56$, and 666.94 for $\mathrm{O}-\mathrm{H}$ stretching, $\mathrm{C}-\mathrm{H}$ stretching, $\mathrm{COOH}$ stretching, $\mathrm{CH}_{2}$ bending, $\mathrm{O}-\mathrm{H}$ bending $\mathrm{C}-\mathrm{O}$ stretching, $\mathrm{C}-\mathrm{H}$ bending, and $\mathrm{C}$-Br stretching, respectively (Fig. 1). The IR spectra of the drug and polymer combination were compared with the spectra of the pure drug and individual excipients, in which no shifting of peaks was significantly found. This indicates no interaction of the drug with other volatile and non-volatile excipients during microballoon formulation development. There were no extra peaks seen other than the normal peak in the spectra of the formulation and thus confirmed the absence of any chemical interaction between the drug and the polymers and they are compatible with each other. These results indicate the method used to prepare microballoons does not affect the physicochemical properties of the systems [23]. The study suggested that acebrophylline can be used with HPMC and EC.

\section{Micrometric properties of microballoons}

The mean particle size of the microballoons formulation MB1 to MB6 containing HPMC and EC was in the range between $226 \pm 17$ and $577 \pm 10$ (Fig. 2a). The mean particle size of the microballoons was found to increase by increasing the polymer concentration. The viscosity of the medium increases at a higher polymer concentration resulting in enhanced interfacial tension. Shearing efficiency is also diminished at higher viscosities [24]. This formation of average particle size was found to be increased. The bulk density and tapped density of formulation MB1 to MB6 ranges from $0.401 \pm 0.02$ to $0.602 \pm 0.01 \mathrm{~g} / \mathrm{cm}^{3}$ and $0.467 \pm 0.02$ to $0.574 \pm 0.03 \mathrm{~g} / \mathrm{cm}^{3}$, respectively (Fig. 2b). The Carr's index ranges between $13.33 \pm 1.6$ and $16.45 \pm 1.9$ (Fig. 2c). The angle of repose of microballoons ranges from $15.12 \pm 1.55$ to $19.35 \pm 2.41$ (Fig. 2d). The values of Carr's index and angle of repose indicate excellent flow properties.

\section{Percentage yield, entrapment efficiency, in vitro buoyancy of microballoons}

Microballoons were weighed after drying, and the percentage yield was calculated [25]. The percentage yield of microballoons containing EC is high when compared with more HPMC-containing microballoons (Fig. 3). Microballoons with a high percentage of HPMC than EC show the percentage yield ranging from $67.76 \pm 0.47$ to $76.87 \pm 1.5 \%$. Microballoons with high EC concentration than HPMC show percentage yield ranging from $89.71 \pm 1.2$ to $97.45 \pm 1.4 \%$. Test for drug content was carried out to ascertain that the drug is uniformly loaded in the formulation. Relatively high entrapment efficiency was observed for all microballoon formulation because the drug was poorly soluble in aqueous medium. The entrapment efficiency ranged between $69.34 \pm 0.33$ and $95.34 \pm 0.54 \%$. It was found that the entrapment efficiency increased with increase in the EC concentration. At the same time, the drug entrapment is decreased when 

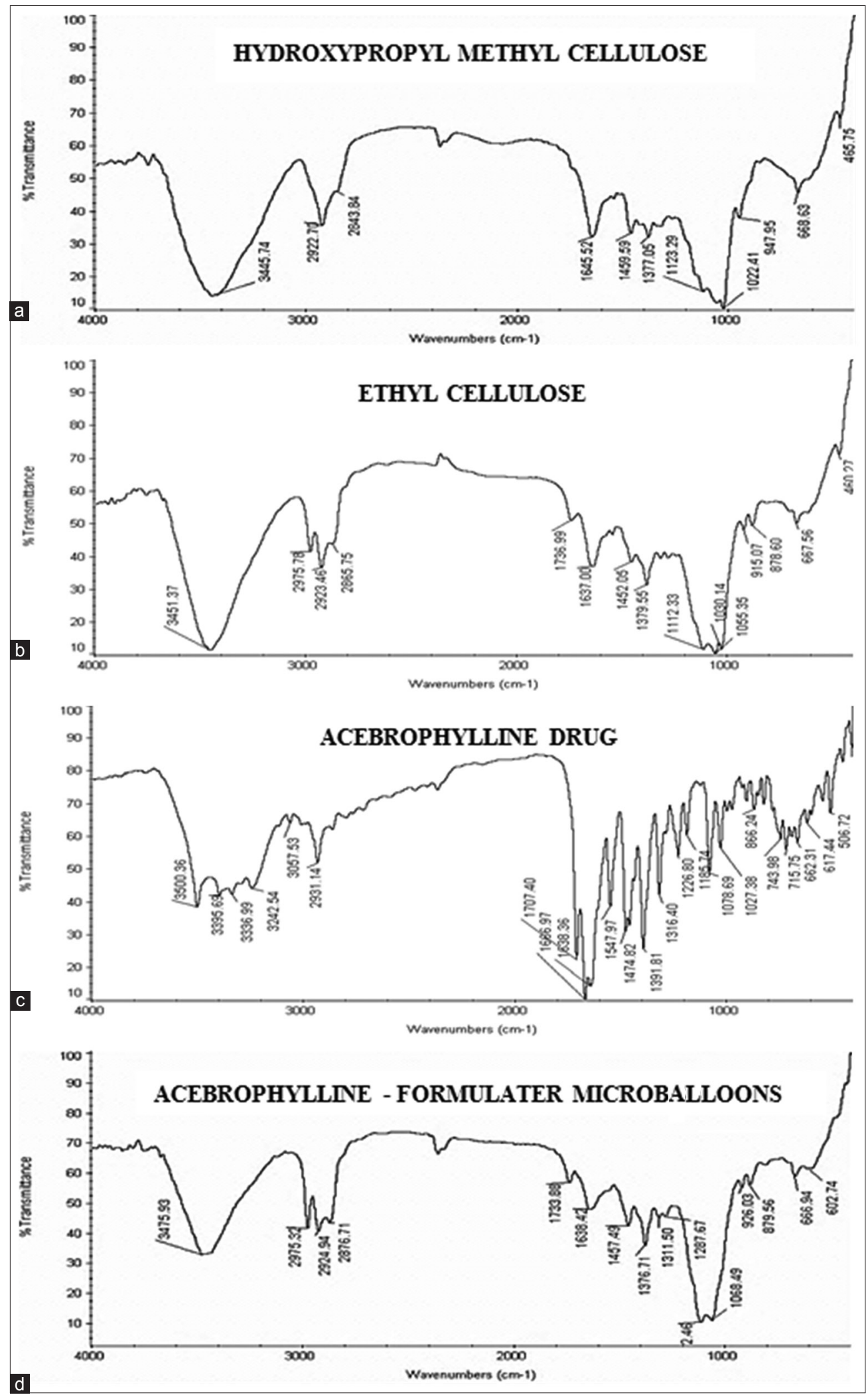

Fig. 1: Fourier transform infrared spectra analysis of drug and polymer compatibility. (a) Hydroxypropyl methylcellulose, (b) ethyl cellulose, (c) acebrophylline drug, (d) acebrophylline - formulater microballoons

there is an increase in the concentration of HPMC. The in vitro buoyancy was carried out to investigate buoyancy of prepared microballoons [26]. The microballoons were spread over the surface of simulated gastric fluid, and the fraction of time was quantitated. The microballoons containing more EC showed good buoyancy when compared to the microballoons containing more HPMC. The microballoons containing a high concentration of HPMC showed buoyancy percentage ranges from $84.71 \pm 1.2$ to $68.57 \pm 0.7 \%$. The microballoons containing high concentration of EC showed buoyancy percentage ranges from $87.91 \pm 1.4$ to $93.67 \pm 1.0 \%$ due to insolubility of EC in gastric fluid ( $\mathrm{pH} 1.2$ ). 

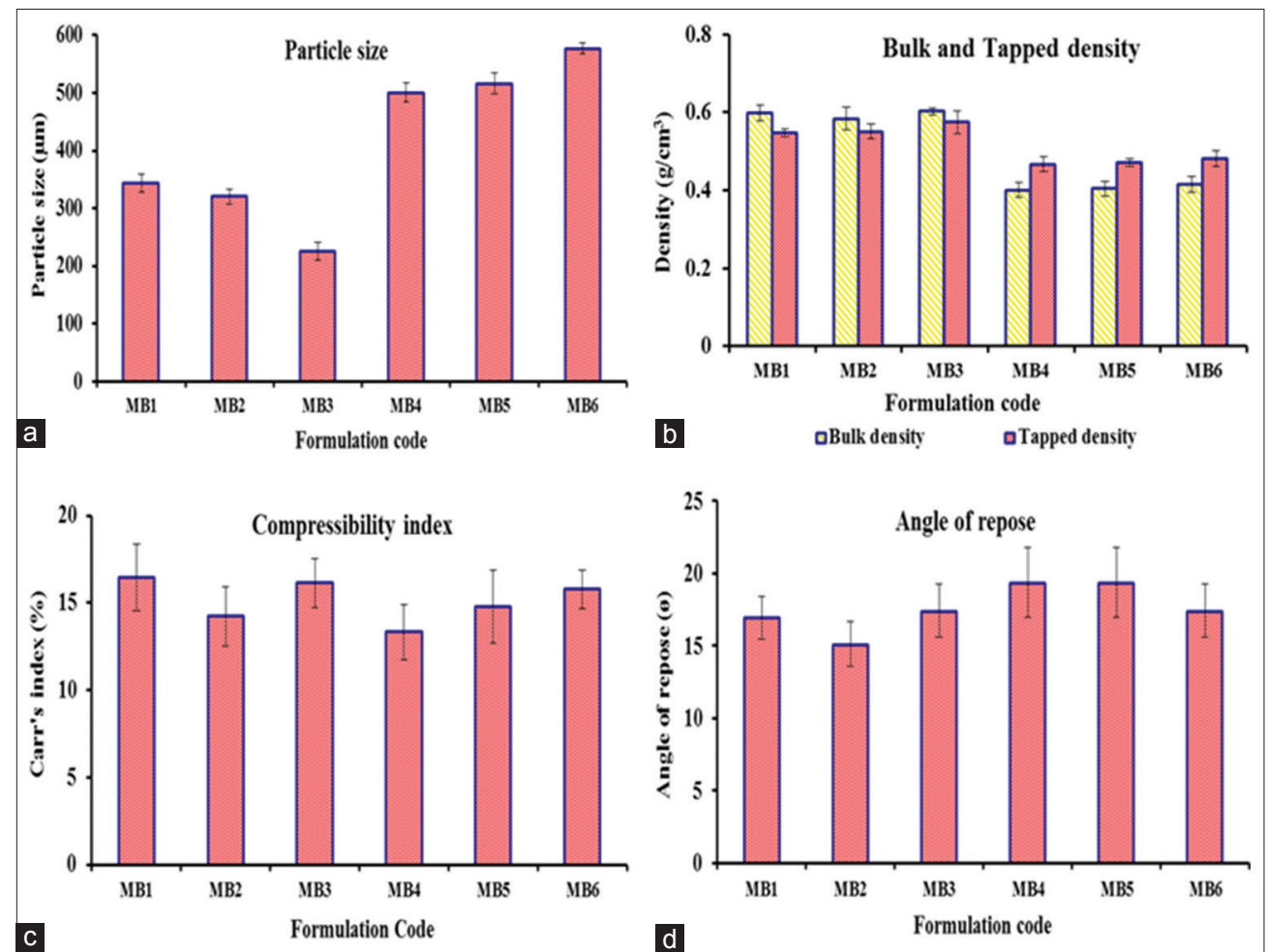

Fig. 2: Micromeritic properties of microballoons. (a) Particle size, (b) bulk and tapped density, (c) compressibility index, (d) angle of response

\section{Surface morphology by SEM}

Morphology of the prepared floating microballoons was examined by SEM. SEM study suggested that hollow microballoons were found to be spherical in shape with smooth texture [27]. Results showed that the view of microballoons clearly indicated that the microballoons were spherical in shape, and there is a formation of a hollow cavity in the sphere. The porous nature and spherical shape were found to be in EC and HPMC-based microballoons. This tendency of the microballoons surface was most probably resulted from the mechanism of solvent evaporation. Microballoons floated more than $12 \mathrm{hrs}$ because of the presence of hollow cavity. The image of the microballoons was shown in (Fig. 4).

\section{Drug release rate}

The drug release of formulations (MB1-MB6) is shown in (Fig. 5). The formulation of MB1, MB2, MB3, MB4, MB5, and MB6 showed percentage drug releases of $89.54 \pm 1.38,66.78 \pm 1.40,52.81 \pm 1.48,89.99 \pm 1.67$, $93.21 \pm 1.57$, and $97.81 \pm 1.79$ at the end of $12 \mathrm{hrs}$, respectively. The increased concentration of polymer increased the density of polymer matrix, which results in increased diffusional path length; this decreased the overall release of drug from polymer matrix [28] The combination of water-soluble polymer and a controlled release polymer in the formulation MB6 produced maximum sustained release of acebrophylline. The in vitro release showed the maximum release of more than $97 \%$ in $12 \mathrm{hrs}$. It was observed that by increasing the concentration of EC alone in a combination of polymer HPMC and EC, the release rate of the drug from the microballoons decreased dramatically.

\section{In vitro drug release kinetics}

The in vitro drug release kinetics mechanism of microballoons was evaluated on the basis of zero order, first order, Higuchi and Peppas models (Fig. 6). The model that best fits the release data are selected based on the value of resulting correlation coefficients $(r)$. The in vitro release data were applied to various kinetic models to predict drug release kinetic mechanism [29]. The zero order plots for all formulation were found linear in acidic medium $\mathrm{pH}$ 1.2. The in vitro drug release

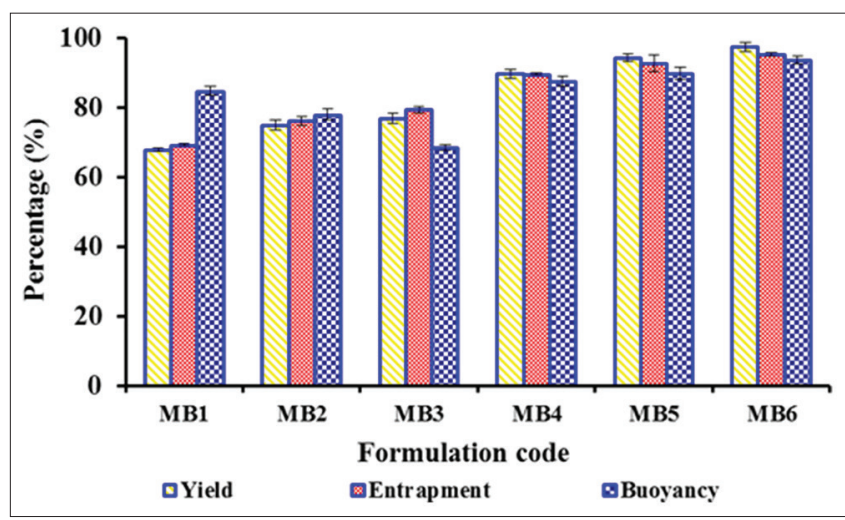

Fig. 3: Percentage yield, entrapment efficiency, in vitro buoyancy of microballoons

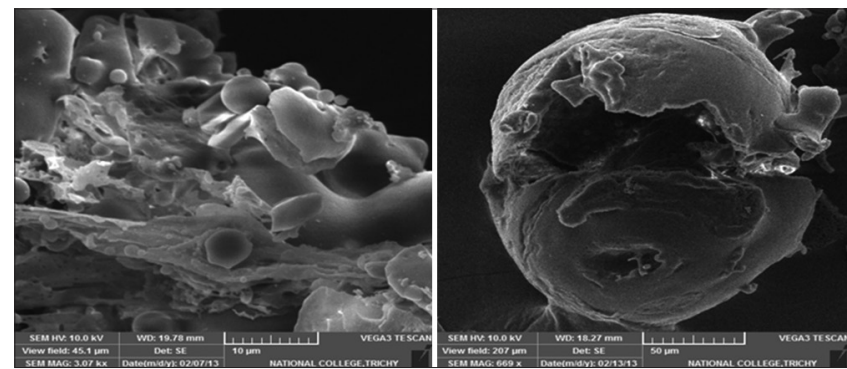

Fig. 4: Scanning electron microphotograph of acebrophylline microballoon

showed the highest regression coefficient values for the zero order model and indicating diffusion to be the predominant mechanism of drug release. 


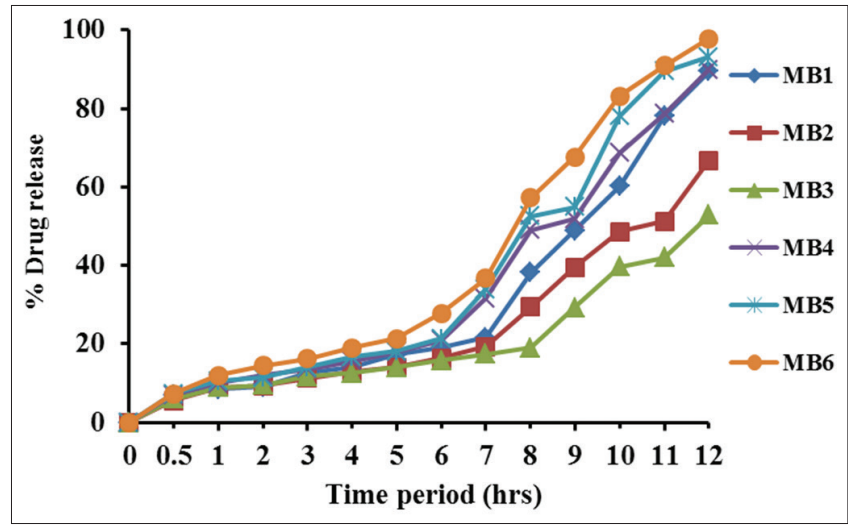

Fig. 5: Comparative in vitro drug release rate of acebrophylline microballoons

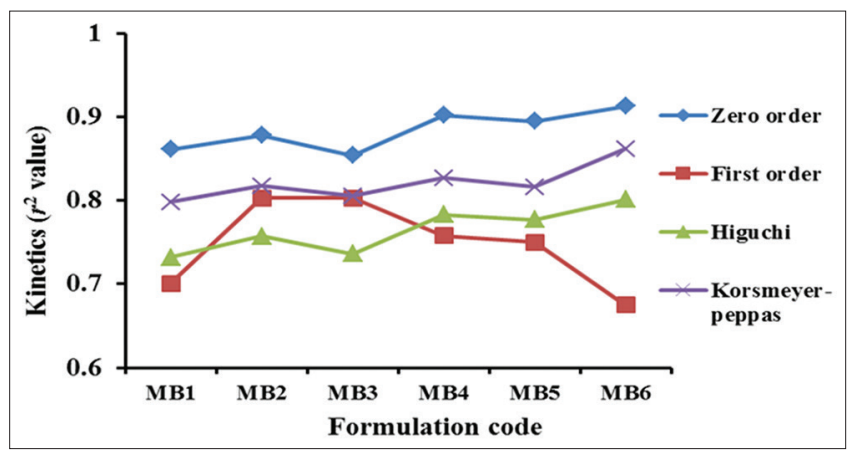

Fig. 6: Drug release kinetics of microballoons

Table 2: Stability studies of microballoons $\left(40^{\circ} \mathrm{C} / 75 \% \mathrm{RH}\right)$

\begin{tabular}{lll}
\hline Days & Physical changes & Percentage entrapment efficiency \\
\hline 0 & - & 97.34 \\
30 & No changes & 97.21 \\
60 & No changes & 96.21 \\
90 & No changes & 95.79 \\
\hline
\end{tabular}

\section{Stability studies}

The stability of preparation is an important factor to estimate the quality of the dosage [30]. The stability study showed that there was no remarkable change in the content of MB6 formulation during 90 days, in which it was stored at $40^{\circ} \mathrm{C}$ and $75 \%$ RH (Table 2).

\section{CONCLUSIONS}

Drug absorption in the gastrointestinal tract is a highly variable process, prolonging gastric retention of the dosage forms, and extending the time of drug absorption. Hollow microballoons, gastroretentive controlled release delivery system promises to be a potential approach for gastric retention. The biocompatible and cost-effective polymers such as EC in combination with HPMC can be used to formulate an efficient floating microparticulate system when it is been formulated in large scale. Hence, the prepared floating microballoons of acebrophylline prove to be a potential candidate for multiple-unit delivery devices adaptable for safe and effective sustained drug delivery.

\section{ACKNOWLEDGMENTS}

The authors are thankful to Sunglow Pharmaceutical Pvt. Ltd., Pondicherry, for providing gift sample of acebrophylline with great pleasure. The authors are also thankful to Periyar College of
Pharmaceutical Sciences, for providing the necessary facilities for research work and to all the staff members for their guidance and help throughout the research work.

\section{REFERENCES}

1. Gupta S, Mali RR, Goel V. Novel study in fast dissolving drug delivery system: A review. Indian J Pharm Biol Res 2015;3(1):93-107.

2. Nayak AK, Maji R, Das B. Gastroretentive drug delivery systems: A review. Asian J Pharm Clin Res 2010;3(1):1-10.

3. Kiss D, Zelkó R. Gastroretentive dosage forms. Acta Pharm Hung 2005;75(3):169-76.

4. Hou SY, Cowles VE, Berner B. Gastric retentive dosage forms: A review. Crit Rev Ther Drug Carrier Syst 2003;20(6):459-97.

5. Kawashima Y, Niwa T, Takeuchi H, Hino T, Itoh Y. Hollow microspheres for use as a floating controlled drug delivery system in the stomach. J Pharm Sci 1992;81(2):135-40.

6. Pujara ND, Patel NV, Thacker AP, Raval BK, Doshi SM, Parmar RB. Floating microspheres: A novel approach for gastro retention. World J Pharm Pharm Sci 2012;1(3):872-95.

7. The Global Asthma Report 2011. Paris, France: The International Union against Tuberculosis and Lung Disease; 2011.

8. Gopinath H, Vedanthan C, Kumar PB. Formulation and evaluation of acebrophylline sustained release matrix tablets. J Chem Pharm Sci 2012;5(2):56-61.

9. Pozzi E. Acebrophylline: An airway mucoregulator and antiinflammatory agent. Monaldi Arch Chest Dis 2007;67(2):106-15.

10. Bauskar MD, Nandedkar SY, Wagh RD. Formulation design and optimization of pulsatile release tablet of acebrophylline with swelling and erodible layers for treatment of nocturnal bronchial asthma. Int J Pharm Sci Res 2011;2(12):3100-8.

11. Streubel A, Siepmann J, Bodmeier R. Multiple unit gastroretentive drug delivery systems: A new preparation method for low density microparticles. J Microencapsul 2003;20(3):329-47.

12. Charulatha R, Damodharan N, Sundaramoorthy R, Abhilash G. Design and evaluation of acebrophylline sustained release matrix tablets. Der Pharm Lett 2012;4(2):530-5.

13. Trivedi P, Verma AM, Garud N. Preparation and characterization of aceclofenac microspheres. Asian J Pharm 2008;2(2):110-5.

14. Martin A, Butamante P, Chun AH. Physical Pharmacy. $4^{\text {th }}$ ed. Philadelphia, PA: Lea \& Febiger; 1993. p. 431.

15. Manavalen R, Ramasamy C. Physical Pharmaceutics. $2^{\text {nd }}$ ed. Tamil Nadu: Vignesh Publisher; 2001. p. 456.

16. Sinha VR, Agrawal MK, Kumria R. Influence of formulation and excipient variables on the pellet properties prepared by extrusion spheronization. Curr Drug Deliv 2005;2(1):1-8.

17. Gattani YS, Bhagwat DA, Maske AP. Formulation and evaluation of intragastric floating drug delivery system of diltiazem hydrochloride. Asian J Pharm 2008;2(4):228-31.

18. USP. The United States Pharmacopoeia XXIV. Rockville, MD: United States Pharmacopoeial Convention; 2000. p. 1941.

19. Sato Y, Kawashima Y, Takeuchi H, Yamamoto H. Physicochemical properties to determine the buoyancy of hollow microspheres (microballoons) prepared by the emulsion solvent diffusion method. Eur J Pharm Biopharm 2003;55(3):297-304.

20. Garg R, Gupta GD. Gastroretentive floating microspheres of Silymarin: Preparation and in vitro evaluation. Trop J Pharm Res 2010;9(1):59-66.

21. Jagtap YM, Bhujbal RK, Ranade AN, Ranpise NS. Effect of various polymers concentrations on physicochemical properties of floating microspheres. Indian J Pharm Sci 2012;74(6):512-20.

22. Garg R, Gupta GD. Progress in controlled gastroretentive delivery systems. Trop J Pharm Res 2008;7(3):1055-66.

23. Mohan Kamila M, Mondal N, Kanta Ghosh L, Kumar Gupta B. Multiunit floating drug delivery system of rosiglitazone maleate: Development, characterization, statistical optimization of drug release and in vivo evaluation. AAPS PharmSciTech 2009;10(3):887-99

24. Reddy P, Dorle AK, Krishna DK. Albumin microspheres: Effect of process variables on the distribution and in vitro release. Drug Dev Ind Pharm 1990;16:1781-803.

25. Hafeez A, Maurya A, Singh J, Rana L. In vitro evaluation of floating microsphere of Ketoprofen. J Sci Innov Res 2013;2(3):714-22.

26. Perumandla PK, Priya S. Formulation and in vitro evaluation of floating microspheres of dextromethorphan hydrobromide. Int J Pharm Pharm Sci 2014;6(4):206-10

27. Tripathi M, Radhika PR, Sivakumar T. Formulation and evaluation of 
glipzide hollow microbaloons for floating drug delivery. Bull Pharm Res 2011;1(1):67-74.

28. Srivastava AK, Ridhurkar DN, Wadhwa S. Floating microspheres of cimetidine: Formulation, characterization and in vitro evaluation. Acta Pharm 2005;55(3):277-85.
29. Dash S, Murthy PN, Nath L, Chowdhury P. Kinetic modeling on drug release from controlled drug delivery systems. Acta Pol Pharm 2010;67(3):217-23.

30. Bajaj S, Singla D, Sakhuja N. Stability testing of pharmaceutical products. J Appl Pharm Sci 2012;2(3):129-38. 